\title{
NOTE ON LOGARITHMIC SWITCHBACK TERMS IN REGULAR AND SINGULAR PERTURBATION EXPANSIONS*
}

\author{
P. A. LAGERSTROM $\dagger \ddagger$ AND D. A. REINELT $\dagger$
}

\begin{abstract}
The occurrence of logarithmic switchback is studied for ordinary differential equations containing a parameter $k$ which is allowed to take any value in a continuum of real numbers and with boundary conditions imposed at $x=\varepsilon$ and $x=\infty$. Classical theory tells us that if the equation has a regular singular point at the origin there is a family of solutions which varies continuously with $k$, and the expansion around the origin has $\log x$ terms for a discrete set of values of $k$. It is shown here how nonlinearity enlarges this set so that it may even be dense in some interval of the real numbers. A $\log x$ term in the expansion in $x$ leads to expansion coefficients containing $\log \varepsilon$ (switchback) in the perturbation expansion. If for a given value of $k$ logarithmic terms in $x$ and $\varepsilon$ occur they may be obtained by continuity from neighboring values of $k$. Switchback terms occurred conspicuously in singular-perturbation solutions of problems posed for semi-infinite domain $x \geqq \varepsilon$. This connection is historical rather than logical. In particular we study here switchback terms for a specific example using methods of both singular and regular perturbations.
\end{abstract}

Formulation of general problem. The customary description of switchback is the following: Assume we have a perturbation problem with a small parameter $\varepsilon, 0<\varepsilon<1$, and an assumed sequence of expansion parameters, $\eta_{n}(\varepsilon)$. It may happen that after having computed to order $\eta_{j}(\varepsilon)$ one originally assumes that the next term is of order $\eta_{j+1}(\varepsilon)$; however, one finds it necessary to insert an intermediate term of order $\eta^{*}(\varepsilon)$, $\eta_{j}<\eta^{*}<\eta_{j+1}$. Obviously, this description is subjective; one simply made a wrong guess about which expansion parameters to use, and more careful scrutiny demands the introduction of an intermediate term. A more objective description is this: At a certain stage in the perturbation expansion the neglected terms are formally ${ }^{1}$ of order $\eta_{j+1}$ whereas actually their effect is of order $\eta^{*}$. Here we shall examine perturbation problems whose formulation involves the small parameter $\varepsilon$ but not $\log \varepsilon$ and discuss the mechanism for the appearance of $\log \varepsilon$ in some expansion coefficients. (This may include terms which normally are not called switchback terms, as in the case $k=1$ in the discussion below.) We shall study this phenomenon for a specific problem or, more precisely, for a family of problems depending on a parameter $k$ and whose solutions $u_{k}(x ; \varepsilon)$ depend continuously on $k$. The general lessons to be drawn from the specific family of problems will be discussed at the end of the paper.

A boundary-value problem. The following problem, ${ }^{2}$ introduced by one of the authors (P.A.L.), will be used to illustrate the main ideas of the present paper,

$(1 \mathrm{a}, \mathrm{b}, \mathrm{c}) \quad \frac{d^{2} \cdot u}{d x^{2}}+\frac{k}{x} \frac{d u}{d x}+u \frac{d u}{d x}=0, \quad u(\varepsilon ; \varepsilon)=0, \quad u(\infty ; \varepsilon)=1$.

We restrict ourselves to the range $k \geqq 1$ and real; the reason for the lower bound will be explained later. It will be essential for our discussion that we deal with a class of problems which is continuous in the sense that the parameter $k$ may take values in a continuum of numbers. We expect the solution $u(x ; \varepsilon, k)$ to vary continuously with $k$.

* Received by the editors August 18, 1983.

† Applied Mathematics Department, California Institute of Technology, Pasadena, California 91125.

$\$$ This author gratefully acknowledges the help and hospitality of the Fondation des Treilles.

${ }^{1}$ The concept of formal order is discussed in detail in [LC].

${ }^{2}$ For various reasons the original problem had a more general equation than (1a), see [CFL] and the references given there. For the purposes of this present paper this generality is irrelevant. 
For future use we give here two equivalent formulations of (1). Defining

$$
v=u-1 \text {, }
$$

we rewrite (1) as

$(3 \mathrm{a}, \mathrm{b}, \mathrm{c}) \quad L_{k}[v] \equiv \frac{d^{2} v}{d x}+\frac{k}{x} \frac{d v}{d x}+\frac{d v}{d x}=-v \frac{d v}{d x}, \quad v(\varepsilon ; \varepsilon)=-1, \quad v(\infty, \varepsilon)=0$.

Secondly we introduce

$$
y=x \varepsilon^{-1}, \quad g(y ; \varepsilon)=u(x ; \varepsilon)
$$

and find

$$
(5 \mathrm{a}, \mathrm{b}, \mathrm{c}) \quad M_{k}[g] \equiv \frac{d^{2} g}{d g^{2}}+\frac{k}{y} \frac{d g}{d y}=-\varepsilon g \frac{d g}{d y}, \quad g(1 ; \varepsilon)=0, \quad g(\infty ; \varepsilon)=1 .
$$

It will be seen later that the linear operator $L_{k}[v]$ has a Green's function for the interval $\varepsilon \leqq x<\infty$ which, however, must depend on $\varepsilon$. The operator $M_{k}[g]$ does not have a Green's function for the interval $1 \leqq y<\infty$. The dependence of the Green's function on $\varepsilon$ in the first case and the nonexistence of a Green's function in the second case are essential for the occurrence of switchback terms. ${ }^{3}$

The problem (1) and generalizations thereof have been studied extensively in the literature. We refer in particular to [LC] and to [CFL], both of which give extensive references. In [LC] the cases $k=0,1,2$ are discussed in detail, and the connection with fluid dynamics (not discussed in the present paper) is given. It is emphasized in [LC] that the problem is not singular for $k=0$. For $k=1$ and $k=2$ it is singular in the sense that it may be treated by singular perturbation techniques. It is explained, however, why the outer expansion is uniformly valid after a suitable regrouping of terms. This means that it may also be treated as a regular perturbation problem. ${ }^{4}$ In [CFL] all real values of $k$ are considered. The treatment there is a rigorous ${ }^{5}$ analysis with the aid of an integral equation equivalent to (1). It is found that the problem is not singular for $k<1$. The exact meaning of this is that for $k<1$ the outer limit $(\varepsilon \downarrow 0$, $x$ fixed) is not $u \rightarrow 1$. It will follow that even the regular perturbation technique used here is also not applicable. We shall therefore restrict $k$ to the range $k \geqq 1$.

Two continuous classes of functions defined by integrals. The exponential integral will play an essential role in the perturbation analysis of our problem. Before introducing it we rewrite the familiar formula for the monomial integral in a form suitable for our purpose.

$$
z=z_{k}(x)=\int_{1}^{x} t^{-k} d t=\frac{x^{1-k}-1}{1-k}, \quad \text { where } x>0 .
$$

(The restriction to real positive values of $x$ is sufficient for our purpose and simplifies the writing.) We regard (6) as valid for all values of $k$, although actually we shall only be concerned with real positive values. Obviously, $z_{1}(x)$ is formally given by an indeterminate expression which may be evaluated by continuity as the function obtained

\footnotetext{
${ }^{3}$ Actually we mean a usable Green's function: Each of the two equivalent formulations leads to an iteration scheme with linear nonhomogeneous equations. A usable Green's function must in principle give a solution of each such equation in the standard manner.

${ }^{4}$ The corresponding statement for fluid dynamics was given in [KL].

${ }^{5}$ The attempt at rigor did not prevent a mistake, which was discovered by the nonrigorous methods used here. This mistake does not influence the rest of [CFL]; it will be corrected in the Appendix.
} 
from $z_{k}(x)$ as $k \rightarrow 1$ (from above or below). One then finds that $z_{1}(x)$ is the function customarily ${ }^{6}$ called $\log x$.

The exponential integral is defined by a formula related to (6),

$$
E_{k}(x)=\int_{x}^{\infty} t^{-k} e^{-t} d t
$$

For $k \geqq 1$,

$$
\begin{gathered}
E_{k}(x)=A_{k}+B_{k}+\sum_{j=1}^{\infty} \frac{(-1)^{j} x^{j-k}}{(j-1) !(j-k)}, \quad A_{k}=C_{k}-B_{k}, \\
C_{k}=\int_{0}^{\infty} t^{-k}\left[e^{-t}-\sum_{j=0}^{[k]-1} \frac{(-t)^{j}}{j !}\right] d t, \quad B_{k}=\frac{(-1)^{[k]-1}}{([k]-1) !([k]-k)},
\end{gathered}
$$

where $[k]=$ greatest integer $\leqq k$.

For $k \neq$ integer the introduction of $B_{k}$ is redundant. However, for $k=$ integer the expansion of $E_{k}(x)$ is indeterminate and by continuity we find that $B_{k}$ combines with one of the terms in the sum to form a term $\log x$ and $A_{k}=C_{k}-B_{k}$ tends to the expression given below,

$$
\begin{gathered}
E_{k}(x)=A_{k}+\frac{(-1)^{k} \log x}{(k-1) !}+\sum_{\substack{j=1 \\
j \neq k}}^{\infty} \frac{(-1)^{j} x^{j-k}}{(j-1) !(j-k)} \\
A_{k}=\frac{(-1)^{k}}{(k-1) !}\left(\gamma-\sum_{j=1}^{k-1} \frac{1}{j}\right), \quad k=\text { positive integer. }
\end{gathered}
$$

We obtain (9) from (8) by letting $k$ decrease or increase to integer values. Thus in spite of the discontinuous function [ $k]$ used in $C_{k}$ in $(8), E_{k}(x)$ is still continuous in $k$.

Solution by a regular perturbation method. As mentioned the outer expansion is also a uniformly valid expansion. We may obtain the outer expansion by a perturbation method applied to (3) in which instead of matching with an inner expansion we satisfy the inner boundary condition to some appropriate order (hence the description "regular perturbation method" used above). For historical reasons, not relevant here, we shall call the method "Oseen iteration." The leading term is obtained by solving the (homogeneous) Oseen equation

$$
L_{k}\left(v_{1}\right)=0
$$

the next term satisfies a nonhomogeneous Oseen equation

$$
L_{k}\left(v_{2}\right)=-v_{1}^{\prime} v_{1} \text {, etc. }
$$

We find

$$
u=1+v_{1}+v_{2}+v_{3}+\cdots,
$$

where

$$
\begin{aligned}
& v_{1}=\eta_{1}(\varepsilon) E_{k}(x) \\
& v_{2}=\eta_{2}(\varepsilon) E_{k}(x)+\eta_{1}^{2}(\varepsilon)\left[E_{k}(x) E_{k-1}^{\prime}(x)+2^{2 k-1} E_{2 k-1}(2 x)\right] \\
& v_{3}=\eta_{3}(\varepsilon) E_{k}(x)+\eta_{1}(\varepsilon) \eta_{2}(\varepsilon) v_{3 p}(x ; \varepsilon) .
\end{aligned}
$$

${ }^{6}$ Inverting (6) we find $x=[1+(1-k) z]^{1 /(1-k)}$. The inverse of $z=\log x$, namely $x=\exp z$, is often defined as limit of this formula as $k \rightarrow 1$. 
Here $v_{2}$ is the same function as given in [LC]. It is a solution of $(10 \mathrm{~b}) ; v_{3}, v_{4}$ obey similar nonhomogeneous Oseen equations which may, in principle, be solved with the aid of a Green's function. We require $v_{3 p}$ to be chosen so that for $x$ small its leading terms is $x^{1-k}$. That this is possible can be shown with the aid of the Green's function.

Special case $\boldsymbol{k}=3 / 2$. A partial expansion of the solution is

$$
\begin{aligned}
u\left(x ; \varepsilon, \frac{3}{2}\right) \simeq & 1-\frac{1}{2} \varepsilon^{1 / 2} E_{3 / 2}(x)+\varepsilon\left\{\frac{3}{8} C_{3 / 2} E_{3 / 2}(x)+\frac{1}{4}\left[E_{3 / 2}(x) E_{1 / 2}^{\prime}(x)+4 E_{2}(2 x)\right]\right\} \\
& +\varepsilon^{3 / 2}\left\{c E_{3 / 2}(x)+v_{3 p}(x)\right\}+\cdots .
\end{aligned}
$$

Its value at the inner boundary is

$$
u(\varepsilon ; \varepsilon)=\varepsilon\left[-1+\frac{3}{8} C_{3 / 2}^{2}+\log 2+\log \varepsilon+\gamma-1+2 c+\varepsilon^{1 / 2} v_{3 p}(\varepsilon)\right]+o(\varepsilon) .
$$

Thus the multiplicative constant $c$ in $v_{3}$ has a term strictly of order unity to which has to be added $-\frac{1}{2} \log \varepsilon$. Hence $v_{3}$ contains a switchback term of order $\varepsilon \log \varepsilon$. There are two lessons to be drawn from this. Firstly, logarithmic switchback can occur for noninteger $k$ provided some $E_{l}, l=$ integer, occurs in the solution. In our case the first effect of the nonlinear terms, appearing here as known forcing terms of a linear equation, is to give a term $\sim E_{l}(2 x)$ where $l=2 k-1$ and thus an integer for $k=\frac{3}{2}{ }^{7}$ Secondly, switchback is in principle not tied to the use of matched asymptotic expansions (although as will be seen, it may be easier to spot when such a method is used).

Solution by matched expansions, Stokes iteration, switchback in inner expansions. The inner expansion may be obtained from the uniformly valid expansion just discussed by replacing $x$ by $\varepsilon y$ and expanding in $\varepsilon$ keeping the coordinate $y$ fixed. It can also be obtained by developing $g(y ; \varepsilon)$ as a series in $\varepsilon$ and inserting it into (5a). The first term $g_{0}(y)$ satisfies the equation $M_{k}\left[g_{0}\right]=0$, called the (homogeneous) Stokes equation; the procedure for obtaining subsequent terms will be called a Stokes iteration. The condition at $y=1$ (inner boundary condition) has always to be satisfied. The outer condition has to be replaced by a matching condition with the outer expansion (this may be the same as satisfying the outer boundary condition, but only for a finite number of terms the number of which increases with $k$ ). Thus we obtain an expansion

$$
u(x, \varepsilon) \equiv g(y, \varepsilon)=g_{0}(y)+\varepsilon g_{1}(y)+\cdots
$$

where, however, switchback terms may have to be inserted.

It may be objected that the use of matched asymptotic expansions is unnecessary since a regular perturbation scheme works. However, from a practical point of view, it may simplify computations. The term $g_{0}(y)$ need only be matched with the first term in the outer expansion, namely $u=1+o(1)$; analogous statements are valid for higher order terms. In a practical problem one is often especially interested in the behavior of solution near the boundary, for instance $(d u / d x)_{x=\varepsilon}$, for which an approximation is obtained from the inner expansion.

The terms $g_{j}(y)$ obey the equation

$$
\frac{d}{d y}\left(y^{k} \frac{d g_{j}}{d y}\right)=-y^{k} r_{j}(y),
$$

where

$$
r_{0}(y)=0, \quad r_{j}(y)=\sum_{m=0}^{j-1} g_{m}(y) \frac{d g_{j-1-m}}{d y}, \quad \text { for } j>0,
$$

\footnotetext{
${ }^{7} \mathrm{~A} \log$ term may occur in $v_{3 p}(x)$. This would give rise to a coefficient $\varepsilon^{3 / 2} \log \varepsilon$ which would show up at a later stage of the expansion (13).
} 
assuming that no log terms appear. We shall call (16) nonhomogeneous Stokes equations, and the process of successively finding the terms in (15) a Stokes iteration. The leading term obeys a homogeneous Stokes equation and is

$$
g_{0}=\left(1-y^{1-k}\right) \text {. }
$$

This term satisfies the inner and outer boundary conditions for $k>1$. A particular solution of the equation for $g_{1}$ is

$$
g_{1 p}=(1-k)\left(\frac{y^{2-k}}{2-k}-\frac{y^{3-2 k}}{(3-2 k)(2-k)}\right),
$$

and the general solution of the homogeneous equation is

$$
g_{1 h}=A y^{1-k}+B \text {. }
$$

The first term in (17b) diverges at infinity for $k<2$, and tends to zero at infinity for $k>2$. For the limiting case $k=2$ we may choose $A$ and $B$ suitably so that $g_{1 p}+g_{1 k}$ tends to

$$
g_{1 p}=-\log y-\frac{\log y}{y}
$$

as $k \rightarrow 2$. As $k \rightarrow \frac{3}{2}$, the second term in $g_{1 p}$ with a suitable $g_{1 h}$ added also gives a term $\sim \log y$ and a switchback term is needed.

Summarizing: For $1<k \leqq 2, g_{1 p}$ diverges at infinity but only the values $k=\frac{3}{2}$ and $k=2$ gives a logarithmic divergence, which then necessitates a switchback term. It is natural to guess that for $2<k \leqq 3, g_{2}$ cannot be made to satisfy the boundary conditions. This is easily checked, furthermore it is seen that logarithmic divergence in $g_{2}$ occurs only for $k=\frac{5}{3}$ and $k=3$.

More generally, it may be derived from (16) that logarithmic divergence and hence switchback will occur for all rational values of $k \geqq 2$ and for all values of the form $k=2-1 / m, m=$ integer $>1$. In general, the term $g_{j}(y)$ has a logarithmic term for

$$
k=\frac{j+m}{m}, \quad m=1,2, \cdots, j+1 \text {. }
$$

The following table shows the values of $k$ for which the $g_{j}$ is the first term of inner expansion giving rise to switchback,

$$
\begin{aligned}
j & =1, & k & =2, \frac{3}{2}, \\
j & =2, & k & =3, \frac{5}{3}, \\
j & =3, & k & =4, \frac{5}{2}, \frac{7}{4}, \\
j & =4, & k & =5, \frac{7}{3}, \frac{9}{5}, \\
j & =5, & k & =6, \frac{7}{2}, \frac{8}{3}, \frac{9}{4}, \frac{11}{6}, \quad \text { etc. }
\end{aligned}
$$

For example, for $k=2$, the first term which needs to be corrected for a switchback term is $g_{1}$. The term $g_{2}$ also needs a correction since $k=(2+2) / 2=2$, etc.

The main lesson to be drawn from the above is that logarithmic terms will eventually occur, for the rational values of $k$ described, that is for practically all rational numbers greater than one.

For $k=\frac{3}{2}$ the nonlinear terms in the Oseen iteration gave rise to the term $\varepsilon E_{2}(2 x)$ in $v_{2}$ as seen from (13). Expanding this term of the regular expansion we find a term $\sim \varepsilon \log x=\varepsilon \log y+\varepsilon \log \varepsilon$ in the inner expansion. Thus an $\varepsilon \log y$ in the inner 
expansion indicates an exponential integral with an integer index in the outer expansion. Conversely this term necessitates a term $\sim \log y$ and also a term $\sim \varepsilon \log \varepsilon$, i.e. switchback in the inner expansion. Thus (19) and (20) indicate when to expect a $\log \varepsilon$ in the inner and in the uniformly valid (outer) expansion. In either case the log terms come from the nonlinear term in the full equation. Stokes iteration is much easier to compute than Oseen iteration and gives a fast way of indicating the occurrence of log terms. For instance, the switchback in the inner expansion occurs in $g_{1}$ for $k=2$ and $k=\frac{3}{2}$. The partial inner expansion $g_{0}+\varepsilon g_{1}$ matches with $1+v_{1}+v_{2}$. However, in the outer expansion (which is actually uniformly valid) switchback occurs for the first time in $v_{3}$ for the case $k=\frac{3}{2}$. In that sense switchback comes earlier in the inner expansion.

Switchback and Green's functions. In [LC] it was pointed out that the occurrence of logarithmic switchback terms implies the nonexistence of a Green's function usable for the Stokes iteration. The domain of integration is $1 \leqq y<\infty$, hence a Green's function would have to be independent of $\varepsilon$ and the effect of a forcing term (independent of $\varepsilon$ ) of a nonhomogeneous Stokes equation would be of exactly the order formally indicated. On the other hand, the Oseen iteration (regular perturbation method) may be carried out with the aid of a Green's function which has to depend on $\varepsilon$ since the domain is $\varepsilon \leqq x<\infty$. In fact, the Green's function is

$$
g(x \mid \xi)= \begin{cases}\frac{E_{k}(\xi)\left[E_{k}(\varepsilon)-E_{k}(x)\right]}{E_{k}(\varepsilon) E_{k}^{\prime}(\xi)}, & \varepsilon \leqq x \leqq \xi, \\ \frac{E_{k}(x)\left[E_{k}(\varepsilon)-E_{k}(\xi)\right]}{E_{k}(\varepsilon) E_{k}^{\prime}(\xi)}, & \xi \leqq x<\infty .\end{cases}
$$

This Green's function is always usable, i.e. its use in the Oseen iteration leads to convergent integrals.

With the aid of the Green's function, we can formulate our problem as an integral equation. In [CFL] a different integral equation was used whose solution by iteration turned out to be very useful. It will be indicated that the straightforward iteration of the integral equation based on the Green's function is related to the Oseen iteration. We may write the iteration scheme as

$$
L\left[v^{(n+1)}\right]=-\frac{d v^{(n)}}{d x} v^{(n)}, \quad v^{(0)}=0,
$$

or explicitly, using (21) and rearranging the terms slightly,

$$
\begin{aligned}
v^{(n+1)}(x)= & -\frac{E_{k}(x)}{E_{k}(\varepsilon)}-\frac{E_{k}(x)}{E_{k}(\varepsilon)} \int_{\varepsilon}^{\infty}\left[E_{k}(\varepsilon)-E_{k}(\xi)\right] \frac{v^{(n)}(\xi) v^{(n)}(\xi)}{E_{k}^{\prime}(\xi)} d \xi \\
& +\int_{x}^{\infty}\left[E_{k}(x)-E_{k}(\xi)\right] \frac{v^{(n)}(\xi) v^{(n)}(\xi)}{E_{k}^{\prime}(\xi)} d \xi, \quad v^{(0)}=0
\end{aligned}
$$

For $n=2$ this gives,

$$
\begin{aligned}
v^{(2)}(x)= & -\frac{E_{k}(x)}{E_{k}(\varepsilon)}-\frac{E_{k}(x)}{E_{k}^{3}(\varepsilon)} \int_{\varepsilon}^{\infty}\left[E_{k}(\varepsilon)-E_{k}(\xi)\right] E_{k}(\xi) d \xi \\
& +\frac{1}{E_{k}^{2}(\varepsilon)} \int_{x}^{\infty}\left[E_{k}(x)-E_{k}(\xi)\right] E_{k}(\xi) d \xi=v_{1}+v_{2} .
\end{aligned}
$$

If we replace $E_{k}(\varepsilon)^{-1}$ by its leading terms for $\varepsilon$ small the $v^{(2)}(x)$ as given above yields $v_{1}+v_{2}$ of (13) for $k=\frac{3}{2}$. In general, the iteration scheme proposed above is related to what we here called the Oseen iteration since $\sum_{j=1}^{n} v_{j}$ can be obtained from $v^{(n)}$. 


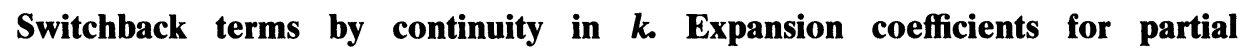
expansions. We have seen that the occurrence of a $\log x$ in the expansion of $u(x ; \varepsilon, k)$ for small values of $x$ leads to switchback terms in the expansion of the function for small values of $\varepsilon$; the reason is simply that an inner boundary is imposed at $x=\varepsilon$. We shall therefore sometimes use the expression "logarithmic terms," without specifying whether $\log x$ or $\log \varepsilon$ is intended. In a standard table of integrals $\log x$ appears abruptly among the integrals of monomials. However, if we use the definite integral (6) $z_{k}$ is continuous at $k=1$ and $z_{1}(x)$ happens to be $\log x$. Similarly, $E_{k}(x)$ is continuous in $k$. The complete expansion for small values of $x$ is known; the terms can be arranged in a special way (see (8) and (9)) to exhibit the occurrence of logarithmic terms by continuity in $k$. We believe $u(x ; \varepsilon, k)$ to be continuous in $k$. However, rigorous demonstrations are not the purpose of this paper, and we shall instead discuss some suitable choices of the expansion coefficients. One extreme is to choose the $\eta_{j}(\varepsilon)$ in (12) in such a way that the inner boundary condition is exactly satisfied. This, however, is unwieldy and the accuracy is spurious since we satisfy the boundary conditions exactly but the equations are not exact. The other extreme is exhibited in (13) which uses the simplest possible form of $\eta_{j}(\varepsilon)$. It demonstrates how logarithmic terms occur for $k=\frac{3}{2}$, but not the continuity in $k$. We shall take an intermediate course: In satisfying the inner boundary condition we use partial expansions of $E_{l}(\varepsilon)$ for $\varepsilon$ small. For $1 \leqq k \leqq 2$ and $k$ irrational we try the form

$$
\begin{aligned}
u(\varepsilon ; \varepsilon)=0= & +\eta_{1}(\varepsilon)\left[\frac{-\varepsilon^{1-k}}{1-k}+A_{k}+B_{k}+\frac{\varepsilon^{2-k}}{2(3-k)}+O\left(\varepsilon^{3-k}\right)\right] \\
+ & \eta_{2}(\varepsilon)\left[\frac{-\varepsilon^{1-k}}{1-k}+A_{k}+B_{k}+O\left(\varepsilon^{2-k}\right)\right] \\
+ & \eta_{1}^{2}(\varepsilon)\left[-\varepsilon^{1-k}\left(A_{k}+B_{k}\right)+2^{2 k-1}\left(A_{2 k-1}+B_{2 k-1}\right)\right. \\
& \left.\quad+\varepsilon^{3-2 k}\left(-\frac{1}{1-k}+\frac{4}{3-2 k}-\frac{1}{2-k}\right)+O\left(\varepsilon^{2-k}, \varepsilon^{4-2 k}\right)\right] \\
+ & \eta_{3}(\varepsilon)\left[-\frac{\varepsilon^{1-k}}{1-k}+O(1)\right]+\eta_{1}(\varepsilon) \eta_{2}(\varepsilon) v_{3 p}(\varepsilon ; \varepsilon) .
\end{aligned}
$$

If $k>1$ the first term in the first bracket dominates. However, we do not get the right limit as $k \downarrow 1$ if $A_{k}+B_{k}\left(=C_{k}\right)$ is neglected. We therefore put

$$
\eta_{1}(\varepsilon)\left[\frac{-\varepsilon^{1-k}}{1-k}+C_{k}\right]=-1
$$

or

$$
\eta_{1}(\varepsilon, k)=\frac{1}{\varepsilon^{1-k}(1-k)^{-1}-C_{k}}
$$

As $k \downarrow 1$ we find

$$
\eta_{1}(\varepsilon, 1) \downarrow \frac{1}{\log \varepsilon+\gamma} .
$$

The term $B_{k}$ is needed to produce $\log \varepsilon ; A_{k} \downarrow-\gamma$ and is not essential and could be omitted. If we expand (24a) we get

$$
\eta_{1}(\varepsilon, k)=(1-k) \varepsilon^{k-1}+(1-k)^{2} \varepsilon^{2 k-2} C_{k} .
$$


We therefore write

$$
\begin{array}{cc}
\eta_{1}(\varepsilon, k)=\frac{1}{\log \varepsilon+\gamma}, & k=1, \\
\eta_{1}(\varepsilon, k)=(1-k) \varepsilon^{k-1}, & 1<k .
\end{array}
$$

Here (25a) is not obtained by continuity from (25b), in fact the whole infinite series in (24c) is needed to obtain (25a). However, once we have satisfied ourselves that $\eta_{1}(\varepsilon, 1)$ is obtainable by continuity we restrict ourselves to $k>1$ and use (25b) rather that the unwieldy (24a). The reasoning warns us, though, that the closer $k$ comes to unity the more important the higher order terms in the expansion $(24 \mathrm{c})$ become.

Using (25b), we now consider the range; $1<k \leqq 2$. After a straightforward calculation,

$$
\begin{aligned}
\eta_{2}=\varepsilon^{2 k-2}(1-k)^{2}\{ & A_{k}+B_{k}+\frac{\varepsilon^{2-k}}{2-k} \\
& \left.+(k-1)\left[A_{k}+B_{k}+\varepsilon^{2-k}\left(\frac{1}{1-k}-\frac{4}{3-2 k}+\frac{1}{2-k}\right)\right]\right\} .
\end{aligned}
$$

For $k<2$ we neglect the terms which are $o(1)$ and find $(27 \mathrm{a})$. However, as $k \uparrow 2$ we must keep the $\varepsilon^{2-k}$ terms and find (27b),

$$
\begin{aligned}
& \eta_{2}(\varepsilon, k)=\varepsilon^{2 k-2} k(1-k)^{2}, \quad 1<k<2, \\
& \eta_{2}(\varepsilon, k)=\varepsilon^{2}(2 \log \varepsilon+2 \gamma+1), \quad k=2 .
\end{aligned}
$$

Again (27a) does not yield (27b) by continuity but (26) shows that a continuous dependence on $k$ can be demonstrated by using more complicated expansion coefficients. Note that at this stage $k=\frac{3}{2}$ does not show any peculiarity since $\eta_{2}\left(\varepsilon, \frac{3}{2}\right)=$ $\varepsilon \frac{3}{8} C_{3 / 2}$ follows from (27a) and checks with (13).

However going one order higher, which means including the last line in (23), $\eta_{3}(\varepsilon)=\varepsilon^{3 k-3}\left\{\frac{(1-k)^{2}}{2-k} \varepsilon^{3-2 k}+k(1-k)^{3} C_{k}^{2}\right.$

From this,

$$
\begin{aligned}
& +(1-k)^{3} 2^{2 k-1}\left[A_{2 k-1}+B_{2 k-1}+(2 \varepsilon)^{3-2 k}\left(\frac{-1}{4(1-k)}+\frac{1}{3-2 k}-\frac{1}{4(2-k)}\right)\right] \\
& \left.+k(1-k)^{4} C_{k} \varepsilon^{k-1} v_{3 p}(\varepsilon ; \varepsilon)\right\} \text {. }
\end{aligned}
$$

$$
\begin{array}{ll}
\eta_{3}(\varepsilon)=\varepsilon^{3 k-3}\left[k(1-k)^{3} C_{k}^{2}+(1-k)^{3} 2^{2 k-1} C_{2 k-1}+D\right], & 1<k<\frac{3}{2} \\
\eta_{3}(\varepsilon)=\varepsilon^{3 / 2}\left[-\frac{1}{2} \ln 2 \varepsilon-\frac{1}{2} \gamma+1-\frac{3}{16} C_{3 / 2}+D\right], \quad k=\frac{3}{2}, & \\
\eta_{3}(\varepsilon)=\varepsilon^{k}\left[\frac{(1-k)^{2}}{2-k}+(1-k)^{3}\left(\frac{-1}{1-k}+\frac{4}{3-2 k}-\frac{1}{2-k}\right)\right], & \frac{3}{2}<k<2 .
\end{array}
$$

Here $D$ is strictly of order unity and the value of the very last term inside the bracket in (29) is $D+o(1)$ (see remark after (12)).

As before (29) is obtained by dropping higher order terms in $(28)$; (28) but not (29), shows continuous dependence on $k$. Since we consider values of $k$ larger than and smaller than $\frac{3}{2}$ we notice a new phenomenon: For $k<\frac{3}{2}, \varepsilon^{3-2 k}<1$ and for $k>\frac{3}{2}$, $\varepsilon^{3-2 k}>1$. This accounts for the different forms of (29a) and (29c). It is typical that 
such a shift in order of a power of $\varepsilon$ occurs at the value of $k$ which gives rise to a $\log \varepsilon$ term. $^{8}$

To summarize: One may choose the $\eta_{j}(\varepsilon)$ to be the "full" expansion coefficients namely those obtained if the exact boundary conditions are satisfied at each stage. These coefficients, and simplified versions thereof, show that the problem is singular (in the sense described earlier) if and only if $k \geqq 1$. This corroborates the corresponding exact statements proved in [CFL]. Note: Using the full expansion coefficients in carrying out the Oseen iteration $n$ times we obtain a partial asymptotic expansion valid for all $k \geqq 1$, and is continuous in $k$. The required logarithmic terms will appear. If simplified versions of the full expansion coefficients are used the relating partial expansions may be valid only for a limited range in $k$. If the simplest versions are used for irrational $k$, that is when the $\eta_{j}(\varepsilon)$ are powers of $\varepsilon$, the log terms cannot be recuperated by continuity.

Classes of problems leading to logarithmic expansion coefficients. The phenomenon of switchback became conspicuous in connection with certain old paradoxes in fluid dynamics whose nature was explained by Kaplun's use of an extended singular perturbation technique, see [LCo], [K1], [PP]. Then log terms started to proliferate and already the first edition of [VanD] contained a section (pp. 200-202) on the "Prevalence of Logarithms." The discussion there is compatible with the one to be given below and the reader is referred to it for further examples.

Clearly, however, log terms are not tied to paradoxes (a term which has only a subjective meaning) or to fluid dynamics. Incidentally, if one restricts oneself to a finite domain rather than a semi-infinite domain the paradoxes (divergence at infinity) disappear but the log terms do not. Finally, as shown above, they are not tied to singular perturbation techniques. They are part of the answer, and the method of using singular perturbations is only one of many possible techniques for arriving at an answer. They do occur only in perturbation expansions, simply because of the way they were defined. We shall now try to describe some general characteristics of perturbation problems whose solution include switchback terms. Earlier we have described the mechanism for the occurrence of log terms in a specific problem. The functions $E_{k}(x)$ contain log terms when $k$ is a positive integer; they thus form a discrete set and have a lower bound. It is convenient in the present case to consider $E_{k}(x)$ as one of the fundamental solutions of the equation $L_{k}[v]=0$. Near the origin the leading term is $\sim x^{1-k}$. For $k<1$ the exponent is greater than zero and subsequent terms are higher (positive) powers of $x$ so that $\log x$ can never appear. From the theory of second-order linear equations with a regular singular point (which we put at the origin) we know that among them is one family of fundamental solutions $F_{k}(x)$ which has logarithmic terms for a discrete set of values of $k$. The functions with logarithmic terms can be obtained by continuity in $k$ (although this is not always emphasized in standard text books). Obviously the $E_{k}(x)$ form a special case. The fact that the equations are of second order is of course irrelevant. The nonlinearity, in a perturbation problem, leads to terms such as $E_{2 k-1}$ discussed above, and may lead to a dense set of values for $k$ for which logarithmic terms appear. Terms such as $E_{2 k-1}$, reminiscent of combination tones arising in the perturbation study of nonlinear oscillator, are actually a general phenomenon. A rational discussion may be possible from the point of view of group representation, see [Vi1], although this subject will not be pursued here Note that

\footnotetext{
${ }^{8} \mathrm{~A}$ related phenomenon actually occurs in (6). Note the necessity here of dealing with functions rather than order classes. On the other hand order classes were essential in Kaplun's resolution of the Stokes paradox, see [K1] and [LG].
} 
occurrence of terms $E_{l}(x), l \neq k$, cannot explain expansion coefficients of the form $\varepsilon^{m} \log ^{n} \varepsilon, n>1$. Obviously (consider e.g. the inner expansions) such terms must occur. A simple concrete example is given in [Lo].

The essence of the occurrence of $\log x$ terms in the solution $F_{k}(x)$ is that it comes from a singular equation, linear or not, and that it has solutions $F_{k}(x)$ which vary continuously with $k$ and which has expansions around $x=0$ involving singular terms the order of which varies with $k$. We have seen the mechanism for the appearance of $\log x$ terms; we have also seen that $\log x$ leads to $\log \varepsilon$ if an inner boundary condition is imposed at $x=\varepsilon$, where $\varepsilon$ is the small parameter. This is a very essential point. It is instructive to regard the problem as a spherically symmetric multidimensional problem, the dimension can be any real number. In our case the linear terms give the spherically symmetric Laplacian in $(k+1)$ dimension, and the boundary condition is imposed on the sphere $S_{k}$ of radius $\varepsilon$. An important fact is that the larger $k$ is the smaller the sphere of a given radius. Let everything be imbedded in three dimensions. Then, for $k=2, S_{2}$ is the surface of the sphere in three dimensions; for $k=1, S_{1}$ is the surface of a circular cylinder; for $k=0, S_{0}$ consists of two planes of a distance $2 \varepsilon$ which divide space into two mutually independent regions. As $\varepsilon \downarrow 0, S_{1}$ and $S_{2}$ shrink to a line and point respectively; $S_{0}$ does not shrink but changes position which is just a shift of the coordinate origin (we need consider only one region in space), and the outer limit is not $u \rightarrow 1$. When the surface shrinks to a line or a point, the outer limit is $u=1$. For $\varepsilon$ small the cylinder has a much greater effect than the sphere which accounts for the fact that the inner solution diverges at infinity. Another effect of the size of $S_{k}$ is this: If the solution has to pass from $u=0$ at $x=\varepsilon$ to a finite value for $x$ large, then the smaller the sphere is the greater the derivative at $x=\varepsilon$ has to be, thus the leading term starts with large singularities. Mathematically, this is illustrated by the fundamental solution of Laplace's equation in its dependence on $k$.

We believe this to be a general situation. We have a body of surface $S$ whose size for a given $\varepsilon$ decreases as $k$ increases. As $\varepsilon$ tends to zero the surface shrinks to an object (point, line) which has no influence on the surrounding medium. In our case this does not apply to $k=0$ which is intuitively obvious and more generally not for $k<1$ (which can be guessed from a simple calculation) but it applies for $k \geqq 1$. In that case the outer limit is a constant and an iteration scheme can be used to solve the problem. The equation for the first term is linear and homogeneous and its expansion around the origin has a singularity whose order increases with $k$.

A physical example is the classical example of low Reynolds number flow past a body. For other examples we refer to [VanD] and to an interesting treatment of exterior problem of height of the meniscus in [Lo].

We shall not discuss here the use of fractional dimensions in studying partial differential equations nor the question whether one can cut between logarithms (see [VanD, 1975, Note 4]).

Appendix I. A correction to [CFL]. In doing the calculations for the case $k=\frac{3}{2}$ as shown above it was noticed that the results contradicted [CFL, Thm. 4.3], and a mistake in the proof given there was discovered. Following the notation in [CFL] we let $u^{*}(x)$ be the exact solution of $(1)$ and $w^{*}(x)$ a solution of (1a) with the term $u d u / d x$ omitted (Stokes equation) with the initial conditions $w^{*}(\varepsilon)=0, d w^{*} / d x=$ $d u^{*} / d x=M^{*}$ at $x=\varepsilon$. In other words $w^{*}$ is a solution of the Stokes equation satisfying the inner boundary condition and having the derivative with respect to $x$ at the inner boundary equal to that of the exact solution. It is easily seen the $w^{*}(x)>u^{*}(x)$ for 
$x>\varepsilon$. Theorem 4.3 of [CFL, p. 203] should be corrected to (we change only the relevant part),

$$
\begin{aligned}
& 1<k<2 \Rightarrow w^{*}(x)-u^{*}(x) \leqslant \varepsilon^{k-1}, \\
& k=2 \Rightarrow w^{*}(x)-u^{*}(x) \leqslant \varepsilon|\log \varepsilon|, \\
& 2<k \Rightarrow w^{*}(x)-u^{*}(x) \leqslant \varepsilon .
\end{aligned}
$$

In [CFL] the bound in (A.1a) was $\varepsilon$ rather than the $\varepsilon^{k-1}$ used here. Actually the correction just shown gives the bound a satisfying monotone dependence on $k$. The break at $k=2$ is due to the switchback term in $g_{1}$. The switchback in $g_{1}$ for $k=\frac{3}{2}$ causes no break since $\varepsilon \log \varepsilon<\varepsilon^{1 / 2}$. If instead of $w^{*}(x)$ we had used an iteration on the Stokes equation, corresponding for example to computing $g_{1}$, with the correct inner boundary conditions and the derivative $M^{*}$ at $x=\varepsilon$, other switchback terms would have caused breaks in the estimates corresponding to (A.1).

The corrected form of [CFL, Thm. 4.3] is valid for the general equation, but here we shall consider only (1). The terms of outer expansion computed above are then uniformly valid. We find for $k=\frac{3}{2}$

$$
\left(\frac{d u}{d x}\right)_{x=\varepsilon}=M^{*}=\frac{1}{2 \varepsilon}\left[1-\frac{1}{2} \varepsilon^{1 / 2} C_{3 / 2}+\varepsilon \log \varepsilon+O(\varepsilon)\right] .
$$

Furthermore, $w^{*}(x)=2 \varepsilon M^{*}\left[1-(\varepsilon / x)^{1 / 2}\right]$. The difference $w^{*}-u^{*}$ is largest at $x=\infty$ where we find

$$
w^{*}(\infty)-u^{*}(\infty)=2 \varepsilon M^{*}-1=-\frac{1}{2} \varepsilon^{1 / 2} C_{3 / 2}+\varepsilon \log \varepsilon+O(\varepsilon) .
$$

(Note that $C_{3 / 2}<0$.) This checks with the new version of Theorem 4.3.

The existence of a switchback term for $k=\frac{3}{2}$ indicated that the original version of Theorem 4.3 might be wrong, and our more detailed computations above indicated that in (A.1) the bound for the estimate should be $\varepsilon^{1 / 2}$. The error in [CFL] is in (b) on p. 203. Actually we only need the value of the integral for $x=\infty$. For $1<k<2$ we find, cf. (9),

$$
\int_{\varepsilon}^{\infty} s^{-k}\left(1-e^{-s}\right) d s=\frac{1}{k-1} \varepsilon^{1-k}-E_{k}(\varepsilon) .
$$

Using the argument in [CFL] one proves (A.1a). This is in agreement with (A.2) which, however, carries the expansion further (although it is not a rigorous estimate) and includes the $\varepsilon \log \varepsilon$ term $\left(<\varepsilon^{1 / 2}\right)$ for $k=\frac{3}{2}$.

\section{REFERENCES}

[CFL] D. S. Cohen, A. FokAs And P. A. LAGerstrom, Proof of some asymptotic results for a model

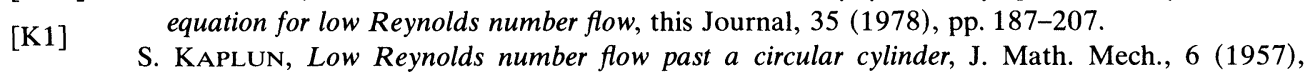
pp. 595-603; reprinted in [K2].

[K2] - Fluid Mechanics and Singular Perturbations, P. A. Lagerstrom, L. N. Howard and C. S. Liu, eds., Academic Press, New York, 1967.

[KL] S. KAPlun AND P. A. LAgERSTrom, Asymptotic expansions of Navier-Stokes solutions for small Reynolds numbers, J. Math. Mech., 6 (1957), pp. 585-593.

[LC] P. A. LAGERSTROM AND R. G. CASTEN, Basic concepts underlying singular perturbation techniques, SIAM Rev., 14 (1972), pp. 63-120. 
[LCo] P. A. LAGERSTROM AND J. D. Cole, Examples illustrating expansion procedures for the NavierStokes equations, J. Rational Mech. Anal., 4 (1955), pp. 817-882.

[Lo] L. L. Lo, The meniscus on a needle-a lesson on matching, J. Fluid Mech., 132 (1983), pp. 65-78.

[PP] I. Proudman AND J. R. A. PEARson, Expansions at small Reynolds numbers for the flow past a sphere and a circular cylinder, J. Fluid Mech. (1957), pp. 237-262.

[VanD] M. VAN DYKe, Perturbation Methods in Fluid Mechanics, Parabolic Press, Stanford, CA, 1975 (Revision of 1964 edition, Academic Press).

[Vi1] N. J. VILENKIN, Special functions and the theory of group representations, AMS Trans. Math. Monographs, 22, American Mathematical Society, Providence, RI. 